\title{
The Right to Effective Behavioral Treatment
}

\author{
Ron Van Houten, Chair \\ Mount Saint Vincent University \\ Saul Axelrod \\ Temple University \\ Jon S. Bailey \\ Florida State University \\ Judith E. Favell \\ Au Clair Program, Mount Dora, Florida \\ Richard M. Foxx \\ Anna Mental Health Center, Anna, Illinois \\ Brian A. Iwata \\ University of Florida \\ O. Ivar Lovaas \\ University of California at Los Angeles
}

\begin{abstract}
We propose that individuals who are recipients or potential recipients of treatment designed to change their behavior have the right to: (1) a therapeutic environment, (2) services whose overriding goal is personal welfare, (3) treatment by a competent behavior analyst, (4) programs that teach functional skills, (5) behavioral assessment and ongoing evaluation, and (6) the most effective treatment procedures available.
\end{abstract}

Over the last several decades, a number of clinical procedures derived from experimental and applied behavior analysis have been developed, evaluated, and refined. These procedures have the demonstrated ability to teach new behavior and alleviate a variety of behavioral disorders. Unfortunately, many who would benefit from behavioral treatment are not receiving it. Behavior analysts have a professional obligation to make available

Report of the Association for Behavior Analysis (ABA) Task Force on the Right to Effective Treatment. Ron Van Houten served as the Task Force Chair and Brian A. Iwata served as the Council Liaison. This report was accepted by the ABA Executive Council; however, it does not necessarily reflect the view of the majority of $A B A$ members nor does it constitute official ABA policy. This report is also published in the Journal of Applied Behavior Analysis (1988), Vol. 21. Reprints may be obtained from Brian Iwata, University of Florida, Gainesville, FL 32611. the most effective treatment that the discipline can provide. Toward this end, the following statement of clients' rights is offered to direct both the ethical and appropriate application of behavioral treatment.

\section{An Individual Has a Right to a Therapeutic Environment}

A physical and social environment that is safe, humane, and responsive to individual needs is a necessary prerequisite for effective treatment. Such an environment provides not only training, but also an acceptable living standard. The dimensions of an adequate living environment are complex and varied; nevertheless, several elements appear essential. Individuals should have access to therapeutic services, leisure activities, and materials that are enjoyable as well as instructive. Thus, client preference, in 
addition to factors such as age-appropriateness and educative value, is relevant in the selection of activities and materials. An adequate environment also includes parents, teachers, and staff who are competent, responsive, and caring. Such qualities may be characterized in terms of frequent positive interactions that are directed toward enjoyment, learning, and independence. Finally, a therapeutic environment imposes the fewest restrictions necessary, while insuring individual safety and development. Freedom of individual movement and access to preferred activities, rather than type or location of placement, are the defining characteristics of a least restrictive environment.

\section{An Individual Has a Right to Services Whose Overriding Goal Is Personal Welfare}

The primary purpose of behavioral treatment is to assist individuals in acquiring functional skills that promote independence. Both the immediate and long-term welfare of an individual are taken into account through active participation by the client or an authorized proxy in making treatment-related decisions. In cases where withholding or implementing treatment involves potential risk, Peer Review Committees and Human Rights Committees play distinct roles in protecting client welfare. Peer Review Committees, comprised of experts in behavior analysis, impose professional standards in determining the clinical propriety of treatment programs. Human Rights Committees, comprised of consumers, advocates, and other interested citizens, impose community standards in determining the acceptability of programs and the extent to which a program compromises an individual's basic rights to dignity, privacy, and humane care; appropriate education and training; prompt medical treatment; access to personal possessions, social interaction, and physical exercise; humane discipline; and physical examination prior to the initiation of a program that may affect or be affected by an individual's health status. Professional competence aided by peer and human rights review will insure that behavioral treatment is delivered within a context of concern for client welfare.

\section{An Individual Has a Right to \\ Treatment by a Competent \\ Behavior Analyst}

Professionals responsible for delivering, directing, or evaluating the effects of behavioral treatment possess appropriate education and experience. The behavior analyst's academic training reflects thorough knowledge of behavioral principles, methods of assessment and treatment, research methodology, and professional ethics. Clinical competence also requires adequate practicum training and supervision, including experience with the relevant client population.

In cases where a problem or treatment is complex or may pose risk, individuals have a right to direct involvement by a doctoral-level behavior analyst who has the expertise to detect, analyze, and manage subtle aspects of the assessment and treatment process that often determine the success or failure of intervention. A doctoral-level behavior analyst also has the ability, as well as the responsibility, to insure that all individuals who participate in the delivery of treatment or who provide support services are trained in the methods of intervention, to assess the competence of individuals who assume subsequent responsibility for treatment, and to provide consultation and followup services as needed.

\section{An Individual Has a Right to Programs That Teach \\ Functional Skills}

The ultimate goal of all services is to increase the ability of individuals to function effectively in both their immediate environment and the larger society. Improvement of functioning may take several forms. First, it often will require the acquisition, maintenance, or generalization of behaviors that allow the individual to gain wider access to preferred materials, activities, or social interaction. 
Second, it may require the acquisition of behaviors that allow the individual to terminate or reduce sources of unpleasant stimulation. Third, improved functioning may require the reduction or elimination of certain behaviors that are dangerous or that in some way serve as barriers to further independence or social acceptability. Finally, as a member of society at large, an individual has a right to services that will assist in the development of behavior beneficial to that society.

Decisions regarding the selection of service goals are not based on a priori assumptions of an individual's behavioral potential or limitations. It is conceivable that some goals might be achieved very slowly, that others may be only approximated, and that, in the process of achieving still other goals, it may be necessary to expose the individual to either immediate temporary discomfort (e.g., as in teaching physical exercise as a means of promoting health) or future risk (e.g., as in teaching an individual to cross streets or to drive an automobile). Still, unless evidence clearly exists to the contrary, an individual is assumed capable of full participation in all aspects of community life and to have a right to such participation.

\section{An Individual Has a Right to Behavioral Assessment and Ongoing Evaluation}

Prior to the onset of treatment, individuals are entitled to a complete diagnostic evaluation to identify factors that contribute to the presence of a skill deficit or a behavioral disorder. A complete and functional analysis emphasizes the importance of events that are antecedent, as well as consequent, to the behavior of interest. For example, identification of preexisting physiological or environmental determinants may lead to the development of a treatment program that does not require extensive use of behavioral contingencies.

The initial behavioral analysis is performed in three stages. First, answers to the following types of questions are ob- tained through interview. Is there any circumstance in which the behavior always occurs? Is there any circumstance in which the behavior never occurs? Does the behavior typically occur at certain times of the day? Could the behavior be associated with any form of discomfort or deprivation? Could events following the behavior serve as either positive reinforcement (e.g., attention) or negative reinforcement (e.g., escape from demands)? The second stage of analysis, direct observation of the individual's behavior under varied and relevant circumstances, confirms suspected relationships identified during the interview. Finally, the assessment findings are incorporated into a systematic treatment plan.

Successful intervention requires ongoing evaluation in the form of objective data to determine the effects of treatment, to quickly identify unanticipated problems, and, if necessary, to modify the treatment plan. The behavior analyst maintains accountability and solicits timely input into the decision-making process by sharing these data regularly with all concerned parties.

\section{An Individual Has a Right to the Most Effective Treatment Procedures Available}

An individual is entitled to effective and scientifically validated treatment. In turn, behavior analysts have an obligation to use only those techniques that have been demonstrated by researchers to be effective, to acquaint consumers and the public with the advantages and disadvantages of these techniques, and to search continuously for the most optimal means of changing behavior.

Consistent with the philosophy of least restrictive yet effective treatment, exposure of an individual to restrictive procedures is unacceptable unless it can be shown that such procedures are necessary to produce safe and clinically significant behavior change. It is equally unacceptable to expose an individual to a nonrestrictive intervention (or a series of such interventions) if assessment results or 
available research indicate that other procedures would be more effective. Indeed, a slow-acting but nonrestrictive procedure could be considered highly restrictive if prolonged treatment increases risk, significantly inhibits or prevents participation in needed training programs, delays entry into a more optimal social or living environment, or leads to adaptation and the eventual use of a more restrictive procedure. Thus, in some cases, a client's right to effective treatment may dictate the immediate use of quicker-acting, but temporarily more restrictive procedures.

A procedure's overall level of restrictiveness is a combined function of its absolute level of restrictiveness, the amount of time required to produce a clinically acceptable outcome, and the consequences associated with delayed intervention. Furthermore, selection of a specific treatment technique is not based on personal conviction. Techniques are not considered as either "good" or "bad" according to whether they involve the use of antecedent rather than consequent stimuli or reinforcement rather than punishment. For example, positive reinforcement, as well as punishment, can produce a number of indirect effects, some of which are undesirable.
In summary, decisions related to treatment selection are based on information obtained during assessment about the behavior, the risk it poses, and its controlling variables; on a careful consideration of the available treatment options, including their relative effectiveness, risks, restrictiveness, and potential side effects; and on examination of the overall context in which treatment will be applied.

\section{CONCLUSION}

Behavior analysts have a responsibility to insure that their clients' rights are protected, that their specialized services are based on the most recent scientific and technological findings, that treatment is provided in a manner consistent with the highest standards of excellence, and that individuals who are in need of service will not be denied access to the most effective treatment available. In promulgating the rights described in this document, the field of behavior analysis acknowledges its responsibilities by reaffirming its concern for individual welfare and by prescribing the means by which behavioral treatment can be delivered in the most beneficial manner. 